PAPER

\title{
Neurogenic pain relief by repetitive transcranial magnetic cortical stimulation depends on the origin and the site of pain
}

\author{
J-P Lefaucheur, X Drouot, I Menard-Lefaucheur, F Zerah, B Bendib, P Cesaro, Y Keravel, \\ J-P Nguyen
}

J Neurol Neurosurg Psychiatry 2004;75:612-616. doi: 10.1136/jnnp.2003.022236

See end of article for authors' affiliations

.....................

Correspondence to: Dr J-Pl Lefaucheur, Service de Physiologie-

Explorations

Fonctionnelles, Hôpital Henri Mondor, 51 avenue de Lattre de Tassigny, 94010 Creteil, France; jean-pascal.lefaucheur@ hmn.ap-hop-paris.fr

Received 30 June 2003

In revised form

13 August 2003

Accepted 23 August 2003

\begin{abstract}
Objective: Drug resistant neurogenic pain can be relieved by repetitive transcranial magnetic stimulation (rTMS) of the motor cortex. This study was designed to assess the influence of pain origin, pain site, and sensory loss on rTMS efficacy.

Patients and methods: Sixty right handed patients were included, suffering from intractable pain secondary to one of the following types of lesion: thalamic stroke, brainstem stroke, spinal cord lesion, brachial plexus lesion, or trigeminal nerve lesion. The pain predominated unilaterally in the face, the upper limb, or the lower limb. The thermal sensory thresholds were measured within the painful zone and were found to be highly or moderately elevated. Finally, the pain level was scored on a visual analogue scale before and after a 20 minute session of "real" or "sham" $10 \mathrm{~Hz}$ rTMS over the side of the motor cortex corresponding to the hand on the painful side, even if the pain was not experienced in the hand itself.

Results and discussion: The percentage pain reduction was significantly greater following real than sham rTMS $(-22.9 \% v-7.8 \%, p=0.0002)$, confirming that motor cortex rTMS was able to induce antalgic effects. These effects were significantly influenced by the origin and the site of pain. For pain origin, results were worse in patients with brainstem stroke, whatever the site of pain. This was consistent with a descending modulation within the brainstem, triggered by the motor corticothalamic output. For pain site, better results were obtained for facial pain, although stimulation was targeted on the hand cortical area. Thus, in contrast to implanted stimulation, the target for rTMS procedure in pain control may not be the area corresponding to the painful zone but an adjacent one. Across representation plasticity of cortical areas resulting from deafferentation could explain this discrepancy. Finally, the degree of sensory loss did not interfere with pain origin or pain site regarding rTMS effects.

Conclusion: Motor cortex rTMS was found to result in a significant but transient relief of chronic pain, influenced by pain origin and pain site. These parameters should be taken into account in any further study of rTMS application in chronic pain control.
\end{abstract}

$\mathrm{D}$ rug resistant neurogenic pain can be treated by precentral motor cortex stimulation with surgically implanted epidural electrodes. ${ }^{1-4}$ Recently, we reported that antalgic effects could also be obtained by non-invasive repetitive transcranial magnetic stimulation (rTMS) of the motor cortex. ${ }^{56}$ Various types of neurogenic pain have been shown to respond to implanted motor cortex stimulation, but such information is lacking for rTMS. Therefore, we studied the influence of the type of lesion at the origin of pain on the effects of rTMS. We also studied the influence of the anatomical site of pain and the degree of sensory loss within the painful zone, as we have recently shown that normal to moderately elevated thermal sensory thresholds within the painful area is associated with a good outcome of the surgical procedure. ${ }^{7}$ We report the results obtained by performing single sessions of $10 \mathrm{~Hz}$ rTMS over the motor cortex in a series of 60 patients with previously intractable, chronic neurogenic pain.

\section{PATIENTS AND METHODS}

\section{Study design}

The study included 60 right handed patients, 32 females and 28 males, aged 27-79 years (mean 54.6 years), suffering from chronic, drug resistant, unilateral neurogenic pain, without past history of seizure. All these patients were referred to evaluate the indication of surgical motor cortex stimulation.
For this purpose, they underwent two tests of rTMS and a quantitative sensory testing in the painful zone and its homologous contralateral side. The study was approved by local and national ethics committees. Two different sessions of rTMS, "real" and "sham", separated by at least 3 weeks were performed in a random order. The patients were not instructed about the existence of a sham stimulation, but were informed that two sessions of rTMS with different parameters of stimulation would be tested for their respective efficacy in relieving pain. This arrangement was made in case some patients experienced a difference between the two sessions, as, from a technical point of view, no ideal sham rTMS could be administered. Although the effects of motor cortex stimulation have been previously found to be somatotopic, ${ }^{2}{ }^{4}$ we decided for the present study to stimulate the motor cortical area corresponding to the hand on the painful side, whatever the site of maximum pain, (face, upper limb, or lower limb). This decision was guided by technical considerations relative to the anatomy of the motor cortex. On the one hand, representation of the lower limbs in the cortex is very medial, at the internal edge of the hemisphere,

Abbreviations: rTMS, repetitive transcranial magnetic stimulation; VAS, visual analogue scale 
quite far from the scalp, and it appeared to us that it was impossible to evoke lower limb motor responses in several patients with neurogenic pain in the lower limb by using a figure of eight coil. On the other hand, representation of the face is very lateral, thus stimulation of the face cortical area induced noticeable facial muscle twitches due to direct stimulation of the ipsilateral facial nerve, even at subthreshold intensity for contralateral cortical evoked motor responses. These rTMS induced twitches allow patients to clearly differentiate between 'real' and 'sham' stimulation. In contrast, sub-threshold stimulation of the hand cortical area induced little local scalp skin sensation, making it difficult for the patients to detect that one of the two sessions, which were separated by at least 3 weeks, was a placebo. These conditions guaranteed that patients were truly blinded to the study.

\section{Origin of pain (type of lesion)}

Five groups of 12 patients were defined regarding the type of lesion at the origin of pain, either a thalamic stroke (infarction or haemorrhage), a brainstem stroke (mesencephalic, pontine, or medullary syndromes), a spinal cord lesion (syringomyelia or post-traumatic ischaemia), a brachial plexus lesion (radiation induced or traumatic), or a trigeminal nerve lesion (failure of trigeminal neuralgia surgery).

\section{Site of pain}

Three groups of patients were defined based on the anatomical site of the pain, The first group (facial pain) comprised 12 patients with trigeminal nerve lesion and 2 patients with brainstem stroke. The second group (upper limb pain) was made up of 12 patients with brachial plexus lesion, 8 patients with thalamic stroke, and 7 patients with brainstem stroke. The third group (lower limb pain) comprised 12 patients with spinal cord lesion, 4 patients with thalamic stroke, and 3 patients with brainstem stroke.

\section{Quantitative sensory testing}

Thermal sensory thresholds were measured over the most painful area of the skin and over the homologous contralateral area. The first perception thresholds for thermal stimulation were measured by heating or cooling the skin using a $16 \mathrm{~cm}^{2}$ Peltier probe and a TSA 2001 apparatus (Medoc, Ramat Yishai, Israel). After an adaptation period at a neutral temperature of $32^{\circ} \mathrm{C}$, temperature was increased (up to $50^{\circ} \mathrm{C}$ ) or decreased (down to $0^{\circ} \mathrm{C}$ ) at a linear rate of $1{ }^{\circ} \mathrm{C} / \mathrm{s}$ until the patient pressed a signal button at the first perception of warm or cold sensation. The thresholds, expressed in ${ }^{\circ} \mathrm{C}$, were calculated as the average value of five consecutive trials. Sensory testing defined two groups of patients; ${ }^{7}$ the first had highly elevated non-nociceptive thermal thresholds in the painful zone, within the range of normal noxious thresholds $\left(>44^{\circ} \mathrm{C}\right.$ for warm threshold and $<20^{\circ} \mathrm{C}$ for cold), while the second group had quite normal to moderately elevated thermal thresholds.

\section{Repetitive transcranial magnetic stimulation}

The two types of rTMS session, real or sham, were identical in their course. The pain was rated by the patient using a $0-10$ visual analogue scale (VAS). The area of the motor cortex corresponding to the hand on the painful side was then determined using the single pulse program of a Super-Rapid Magstim magnetic stimulator (Magstim Co., Whitland, UK) with a figure of eight shaped coil (70 mm double coil 9925-00, Magstim Co) attached to the scalp. This area was identified as the site at which single pulse TMS contralaterally evoked a motor potential of maximal amplitude in the first dorsal interosseus muscle of the hand, ipsilateral to the painful zone. The motor evoked potentials were recorded using a standard EMG machine (Phasis II; EsaOte, Florence, Italy) and surface electrodes. This procedure ensured stimulation over the precentral gyrus. ${ }^{8}$ We then determined the resting motor threshold, defined as the lowest stimulation intensity allowing evocation of motor responses $>50 \mu \mathrm{V}$ peak to peak amplitude in 5/10 trials with the patient at rest. ${ }^{9}$ rTMS was applied using the Super-Rapid Magstim magnetic stimulator with a figure of eight coil centred over the motor cortex area. Following this, one of the following two protocols was applied in a random order: (a) a series of 20 trains of 5 seconds' duration $(55$ second intertrain interval) at a stimulation rate of $10 \mathrm{~Hz}$ and $80 \%$ of resting motor threshold intensity using a real TMS coil; and (b) the same protocol using a sham figure of eight coil (Magstim placebo coil system model 1730-23-00; Magstim Co). Whatever the session, the coil was maintained steady throughout the whole session, tangentially to the scalp, in a postero-anterior direction. Finally, the patients were instructed to rate their pain less than 5 minutes after the rTMS session.

Two methodological points need to be explained: the parameters of stimulation and the sham conditions. Firstly, rTMS parameters for chronic pain have historically been based on the first studies that reported clinical effects of rTMS in patients with depression (20 trains of 10 seconds' duration applied at $10 \mathrm{~Hz}$ frequency). ${ }^{10}$ The frequency of stimulation that we applied ( $10 \mathrm{~Hz}$ ) was close to that used in implanted cortical stimulation for chronic pain $(20-40 \mathrm{~Hz})^{4}$ and was found to be clinically effective (compared with $0.5 \mathrm{~Hz}$ ) in our initial work. ${ }^{5}$ Secondly, the Magstim placebo coil system, which has been designed so as not to have a stimulating effect on the cortex, was preferred to the classical sham condition, which consists of holding a real TMS coil elevated and angled $45^{\circ}$ away from the skull. Tilting the coil off the scalp was found to produce substantial stimulation of the cortex, ${ }^{11}$ particularly when associated with scalp sensation. ${ }^{12}$ Therefore, this arrangement did not meet the criteria for an ideal sham, ${ }^{12}$ which should be performed by means of a coil similar to the real one in shape, weight, and location on the scalp, producing a similar sound and similar scalp skin sensation, but generating no electrical field within the cortex. Such a sham coil has not yet been designed, and at present, the sham coil used in this study is to our knowledge the more valid for clinical trials.

\section{Clinical efficacy}

The percentage of pain level modification was calculated from the VAS scores measured before and after each rTMS session, either real or sham by the following equation:

\section{(post-rTMS-pre-rTMS pain scores $) \times 100 /($ pre-rTMS} pain score).

The result obtained during the sham session was then subtracted from that obtained during the real session to define the true efficacy of rTMS on pain, taking into account the placebo effect.

\section{Statistical analyses \\ Entire series}

The first statistical analysis was performed on the entire series of 60 patients. The VAS scores measured before real and sham rTMS sessions were compared to ensure the reproducibility of these scores at the beginning of each type of session. The VAS scores were then compared before and after the rTMS session, either real or sham, to assess the antalgic efficacy of each type of session. The per cent modification in pain level induced by the real rTMS was 
then compared with that induced by the sham rTMS to evaluate the respective antalgic efficacy of both sessions. All these analyses were performed using a paired $t$ test. Finally, using an unpaired $t$ test, the real-sham \% modification in pain level, as defined above, was compared between the half series of patients who received the real stimulation at the first session and those who received the sham stimulation at this session, to assess the influence of session order on rTMS efficacy. We used parametric tests to analyse results in the entire series of 60 patients by assuming that the data were sampled from Gaussian distributions. We tested this assumption using the Kolmogorov-Smirnov method, which confirmed that the data passed the normality test. In addition, we verified that the pairing of the data was statistically effective before applying a paired $t$ test. A two tailed p value $<0.05$ was considered significant in all cases.

\section{By groups}

A second type of statistical analysis was performed to determine the respective influence of the origin of pain (type of lesion), the site of pain, and the degree of sensory loss within the painful zone on the clinical outcome of the motor cortex rTMS procedure. The patients were divided into five groups according to the type of lesion: thalamic stroke, brainstem stroke, spinal cord lesion, brachial plexus lesion, or trigeminal nerve lesion; into three groups according to the site of pain predominance: face, upper limb, or lower limb; and into two groups according to the thermal thresholds within the painful zone: highly elevated or quite normal. The real-sham \% pain level modifications were compared between the groups defined for pain origin or site with a Kruskal-Wallis non-parametric test and Dunn's post hoc test, and between the groups defined for sensory thresholds with a Mann-Whitney test.

Finally, in each group determined as above, according to one of the three variables (pain origin, pain site, and sensory thresholds), we compared the real-sham \% pain level reduction between all subgroups defined by each one of the other two variables. These analyses were intended to study the influence of the relationship between the three variables on rTMS efficacy and were performed using Kruskal-Wallis or Mann-Whitney tests. As these variables were qualitative and dependent, this statistical approach was more valid than a stepwise multiple regression to compare their respective interest in predicting the clinical outcome. Non-parametric tests were used because data for group analysis were fewer than data for the entire series and did not pass the normality test. A two tailed $\mathrm{p}$ value $<0.05$ was considered significant in all cases.

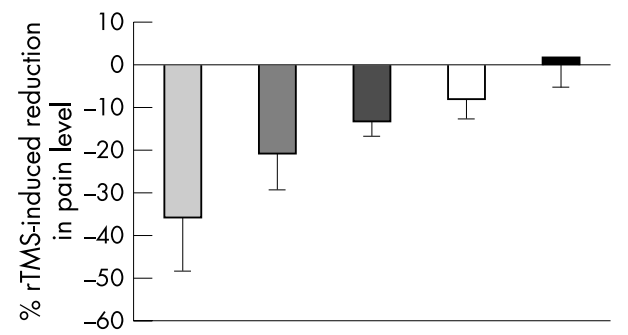

Figure 1 Mean (SEM) \% pain reduction on a visual analogue scale induced by a single session of repetitive transcranial magnetic stimulation of the motor cortex (values calculated by subtracting the results obtained using a sham coil from those obtained using a real coil), depending on the type of lesion at the origin of pain. From left: trigeminal nerve lesion, thalamic stroke, brachial plexus lesion, spinal cord lesion, brainstem stroke. Kruskal-Wallis test, $\mathrm{p}=0.039$.

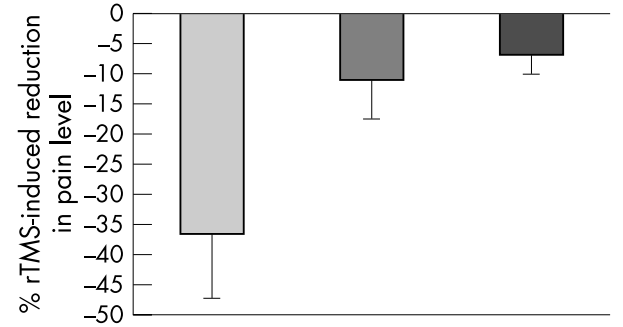

Figure 2 Mean (SEM) \% pain reduction on a visual analogue scale induced by a single session of repetitive transcranial magnetic stimulation of the motor cortex (values calculated by subtracting the results obtained using a sham coil from those obtained using a real coil), depending on the site of pain predominance. From left: face, lower limb, upper limb. Kruskal-Wallis test, $\mathrm{p}=0.022$.

\section{RESULTS}

\section{Overall results}

No adverse effects of rTMS were observed; in particular no seizures were induced. In the entire series of 60 patients, the VAS scores obtained before the real rTMS session were similar to those obtained before the sham rTMS session (6.8 $(0.2) v 6.8(0.2), \mathrm{p}=0.99)$ (mean (SEM), paired $t$ test). The post-rTMS VAS scores were significantly lower than the prerTMS scores for both the real $(5.4(0.3) v 6.8(0.2), \mathrm{p}<0.0001)$ and sham $(6.2(0.3) \vee 6.8(0.2), \mathrm{p}=0.01)$ rTMS sessions. However, the per cent pain reduction was significantly greater following real than sham rTMS $(-22.9 \%$ (3.6) $v$ $-7.8 \%$ (3.2), $p=0.0002$ ). Finally, the real-sham \% pain reduction did not differ between patients who received the real or the sham stimulation at the first session $(-16.1 \%$ (5.3) $v-13.7 \%$ (5.3), unpaired $t$ test, $\mathrm{p}=0.75$ ).

\section{Group results}

Group analyses are illustrated by figs $1-3$ and show that the real-sham \% pain reduction depended on pain origin (Kruskal-Wallis test, $\mathrm{p}=0.039$ ), pain site (Kruskal-Wallis test, $\mathrm{p}=0.022$ ) and sensory loss (Mann-Whitney test, $\mathrm{p}=0.049)$. Dunn's post hoc tests revealed a significant difference $(p<0.05)$ in terms of rTMS efficacy between patients with trigeminal nerve lesion and those with brainstem stroke, and between patients with facial pain and those with upper or lower limb pain.

\section{Individual results}

Pain level was reduced by rTMS in $65 \%$ of the patients $(>30 \%$ in $26.7 \%$ of the patients (good result) and $<30 \%$ in $38.3 \%$ of the patients), while pain level remained unchanged or worsened in $35 \%$ of the patients. Regarding the type of

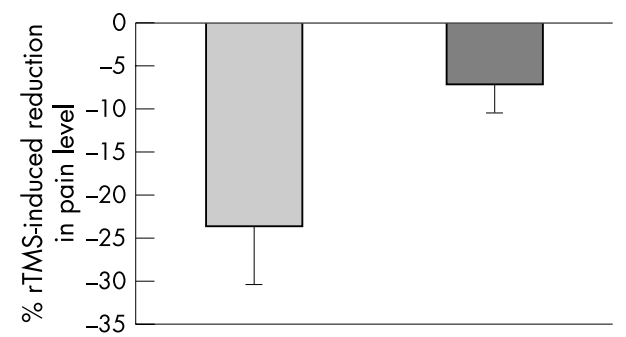

Figure 3 Mean (SEM) \% pain reduction on a visual analogue scale induced by a single session of repetitive transcranial magnetic stimulation of the motor cortex (values calculated by subtracting the results obtained using a sham coil from those obtained using a real coil), depending on the degree of sensory loss within the painful zone: quite normal thermal thresholds (left), highly elevated thresholds (right); MannWhitney test, $p=0.049$. 
lesion, the best results were obtained in patients with trigeminal nerve lesion (good individual results in $58.3 \%$ of these patients), then in patients with thalamic stroke. The worst results corresponded to brainstem strokes. Regarding pain site, more good results were observed in cases of facial pain $(64.3 \%$ of these patients) than in cases of limb pain.

\section{Relation between the different variables}

Regarding the relation between the three variables (pain origin, pain site, and sensory thresholds) and rTMS efficacy, we found using the Kruskal-Wallis test that the origin of pain influenced rTMS results in patients with upper limb pain $(p=0.010)$, and tended to influence rTMS results in patients with severe sensory loss and in patients with lower limb pain $(p=0.053$ and 0.070 , respectively). In all these cases, results were worse in patients with brainstem stroke. The site of pain influenced rTMS results in patients with severe sensory loss $(p=0.025)$ and tended to influence rTMS results in patients with brainstem stroke $(p=0.072)$. In all these cases, patients with facial pain responded better than patients with limb pain. Finally, we did not find any influence of the degree of sensory loss on rTMS efficacy in any subgroup of patients defined by the origin or the site of pain.

\section{DISCUSSION}

This study confirmed that a single session of $10 \mathrm{~Hz}$ rTMS over the motor cortex could reduce pain level in patients suffering from chronic, intractable neurogenic pain. The mean pain level was similar before real or sham rTMS sessions (mean VAS score of 6.8) and session order did not influence rTMS results. Interestingly, a placebo effect was found, as both types of rTMS session resulted in a significant decrease in VAS score. However, real rTMS provoked a greater pain reduction than sham rTMS, attesting to the true efficacy of rTMS on chronic pain. This study also demonstrated that rTMS efficacy was influenced by several clinical variables: pain origin, pain site, and sensory loss within the painful zone.

The most favourable condition was trigeminal nerve lesion, facial pain, and absence of severe sensory loss within the painful zone. The worst condition was brainstem stroke, limb pain, and severe sensory loss. However, it was difficult to delineate the respective influence of these three qualitative and dependent variables on the final result of rTMS procedure. For instance, $86 \%$ of the patients suffering from facial pain presented trigeminal nerve lesion and normal to moderately elevated sensory thresholds. The site of pain was identical within three groups defined by the type of lesion (face for trigeminal nerve lesion, upper limb for brachial plexus lesion, and lower limb for spinal cord lesion). Only patients with thalamic or brainstem stroke could present any type of pain location. By analysing subgroups of patients, it appeared that patients with facial pain tended to respond better than patients with limb pain in cases of brainstem stroke or severe sensory loss. In contrast, patients with brainstem stroke tended to respond worse than patients with pain of other origins, at least in cases of limb pain or severe sensory loss.

Trigeminal neuropathic pain is a favourable condition for surgically implanted motor cortex stimulation. ${ }^{241314}$ Apart from trigeminal nerve lesion, pain location at the face appeared as a positive predictive factor for rTMS efficacy in the present study. The discrepancy between the site of rTMS stimulation (hand cortical area) and the optimal site of pain relief (face rather than upper limb) could be surprising if efficacy of implanted motor cortex stimulation is considered to be somatotopic. ${ }^{24}$

One explanation could result from physiological differences between these two types of stimulation. When the coil is aligned in a postero-anterior orientation as in the present study, tangentially oriented corticocortical fibres should be preferentially stimulated by transcranial magnetically induced cortical currents. ${ }^{15}{ }^{16}$ In contrast, perpendicularly oriented fibres, parallel to the lines of current at the vicinity of the electrodes, should be preferentially stimulated by small epidurally implanted electrodes in a monopolar or bipolar montage. ${ }^{17}$

In addition, rTMS procedure is probably affected by the reorganisation of cortical areas induced by deafferentation in patients with chronic neurogenic pain. In patients with upper limb amputation, the former hand area of the cortex was shown to be invaded by a lateralisation of the area corresponding to arm muscle proximal to the stump, ${ }^{18} 19$ and a medialisation of the face area. ${ }^{1820}$ Similarly, in patients with facial palsy, TMS shows an enlargement of the hand field extending in a lateral direction into the site of the face area. ${ }^{21}$ Additional changes may occur in the cortical output for segments adjacent to the lesion. For instance, a transient ischaemic deafferentation of the hand resulted in an increase of motor cortical output from arm representation. ${ }^{22}$ This motor output was reinforced by low rate $0.1 \mathrm{~Hz}$ rTMS over the arm area, but was reduced when nearby representations were stimulated (hand or face)..$^{23}$ Stimulation of the hand cortical area gave better results in patients with a neurogenic lesion at the face, either because the face area may shift towards the hand area in cases of facial lesion, or because fast rate $10 \mathrm{~Hz}$ rTMS over the hand area could modulate some output from the nearby face cortical representation. Area proximity could not play a role for the lower limbs, as leg and hand representations are not adjacent but separated by arm and trunk representations.

Thalamic pain is also a good indication of surgically implanted motor cortex stimulation. ${ }^{24}{ }^{25}$ The so-called "thalamic syndrome" of Dejerine-Roussy ${ }^{26}$ involves a lesion of the ventrobasal sensory complex of the thalamus, with relatively mild motor deficit, as in our patients with thalamic stroke. In this situation, the ventrolateral motor part of the thalamus is preserved and its activation could be the first step in the pain relieving process induced by motor cortex stimulation, as strongly suggested by functional imaging studies. ${ }^{27}$ We found, using the rTMS procedure, that patients with thalamic strokes benefited from cortical stimulation more than patients with spinal cord lesion or with brainstem stroke. This result argues for a descending modulation triggered by the motor corticothalamic output that could take place in the spinal cord and the brainstem. Increase in cerebral blood flow in the upper brainstem and modulation of nociceptive spinal reflexes during antalgic motor cortical stimulation support the implication of a descending control at these levels. ${ }^{27}$ Various descending modulatory pathways in the brainstem acting on pain transmission have been described. ${ }^{28}$ In this series, patients with brainstem stroke could have damaged these structures, leading to the inefficacy of corticothalamic descending control triggered by rTMS.

In conclusion, even if only less than one third of patients in the whole series experienced good pain relief (reduction of VAS score by more than 30\%) immediately after rTMS session, these results are encouraging for the development of rTMS studies on pain control. Firstly, antalgic effects obtained after a single session of real rTMS were significant compared with placebo in patients suffering from severe chronic pain resistant to all medication. Secondly, selected indications of rTMS according to pain distribution or type of lesion could increase the rate of clinical efficacy; for example, we found more than $60 \%$ of good results occurred in patients with facial pain. Thirdly, our results seem to indicate that the cortical target for rTMS procedure in pain control may not be the area corresponding to the painful zone, in contrast to the 
surgical procedure, and this observation has to be taken into consideration for the design of further pain studies using rTMS. Fourthly, considering that the optimal effect of rTMS on pain is delayed by $2-4$ days, ${ }^{6}$ the immediate pain relief observed in this series could have resulted in more marked effects on the following days, as was experienced and related by some of our patients, but not systematically assessed in the present study. Fifthly, repeated daily sessions of rTMS are able to expand the effects of a single session, as shown in other clinical indications such as severe depression, ${ }^{29}{ }^{30}$ and should be tried for the control of neurogenic pain for a longer period of time. All these observations open exciting perspectives for clinical application of rTMS in pain research, at least in selected patients suffering from chronic neurogenic pain.

\section{ACKNOWLEDGEMENTS}

The work was supported by a grant from the Institut UPSA de la douleur.

\section{Authors' affiliations}

J-P Lefaucheur, X Drouot, I Menard-Lefaucheur, F Zerah, B Bendib, Service de Physiologie-Explorations Fonctionnelles, Hôpital Henri Mondor, Créteil, France

B Bendib, Y Keravel, J-P Nguyen, Service de Neurochirurgie, Hôpital Henri Mondor, Créteil, France

Pierre Cesaro, Service de Neurologie, Hôpital Henri Mondor, Assistance Publique-Hôpitaux de Paris, Paris, France

Jean-Pascal Lefaucheur, Xavier Drouot, Pierre Cesaro, Jean-Paul

Nguyen, INSERM U421, Faculté de Médecine de Créteil, Créteil, France

Competing interests: none declared

\section{REFERENCES}

1 Tsubokawa T, Katayama Y, Yamamoto T, et al. Chronic motor cortex stimulation for the treatment of central pain. Acta Neurochir Suppl (Wien) 1991;52:137-9.

2 Meyerson BA, Lindblom U, Linderoth B, et al. Motor cortex stimulation as treatment of trigeminal neuropathic pain. Acta Neurochir Suppl (Wien) 1993; 58:150-3.

3 Mertens $P$, Nuti $C$, Sindou $M$, et al. Precentral cortex stimulation for the treatment of central neuropathic pain: results of a prospective study in a 20patient series. Stereotact Funct Neurosurg 1999;73:122-5.

4 Nguyen JP, Lefaucheur JP, Decq P, et al. Chronic motor cortex stimulation in the treatment of central and neuropathic pain. Correlations between clinical, electrophysiological and anatomical data. Pain 1999;82:245-51.

5 Lefaucheur JP, Drouot X, Keravel Y, et al. Pain relief induced by repetitive transcranial magnetic stimulation of precentral cortex. NeuroReport $2001 ; 12: 2963-5$.

6 Lefaucheur JP, Drouot X, Nguyen JP. Interventional neurophysiology for pain control: duration of pain relief following repetitive transcranial magnetic stimulation of the motor cortex. Neurophysiol Clin 2001;31:247-52.

7 Drouot X, Nguyen JP, Peschanski M, et al. The antalgic efficacy of chronic motor cortex stimulation is related to sensory changes in the painful zone. Brain 2002; 125:1660-4.
8 Wassermann EM, Wang B, Zeffiro TA, et al. Locating the motor cortex on the $M R I$ with transcranial magnetic stimulation and PET. Neuroimage 1996;3:1-9.

9 Rossini PM, Barker AT, Berardelli A, et al. Non-invasive electrical and magnetic stimulation of the brain, spinal cord and roots: basic principles and procedures for routine clinical application. Report of an IFCN committee. Electroencephalogr Clin Neurophysiol 1994;91:79-92.

10 Pascual-Leone A, Rubio B, Pallardo F, et al. Rapid-rate transcranial magnetic stimulation of left dorsolateral prefrontal cortex in drug-resistant depression. Lancet 1996;347:233-7.

11 Lisanby SH, Gutman D, Luber B, et al. Sham TMS: intracerebral measurement of the induced electrical field and the induction of motor-evoked potentials. Biol Psychiatry 2001;49:460-3.

12 Loo CK, Taylor JL, Gandevia SC, et al. Transcranial magnetic stimulation (TMS) in controlled treatment studies: are some "sham" forms active? Biol Psychiatry 2000;47:325-31.

13 Ebel H, Rust D, Tronnier V, et al. Chronic precentral stimulation in trigeminal neuropathic pain. Acta Neurochir (Wien) 1996;138:1300-6.

14 Nguyen JP, Keravel Y, Feve A, et al. Treatment of deafferentation pain by chronic stimulation of the motor cortex: report of a series of 20 cases. Acta Neurochir Suppl (Wien) 1997;68:54-60.

15 Nakashima H, Kitagawa H, Kawaguchi $Y$, et al. Direct and indirect activation of human corticospinal neurons by transcranial magnetic and electric stimulation. Neurosci Lett 1996;210:45-8.

16 Kaneko K, Kawaii S, Fuchigami Y, et al. The effect of current direction induced by transcranial magnetic stimulation on the corticospinal excitability in human brain. Electroencephalogr Clin Neurophysiol 1996;101:478-82.

17 Comte P. Monopolar versus bipolar stimulation. Appl Neurophysiol 1982;45:156-9.

18 Pascual-Leone A, Peris M, Tormos JM, et al. Reorganization of human cortical motor output maps following traumatic forearm amputation. NeuroReport 1996:7:2068-70.

19 Schwenkreis $\mathbf{P}$, Witscher K, Janssen F, et al. Assessment of reorganization in the sensorimotor cortex after upper limb amputation. Clin Neurophysiol 2001;112:627-35.

20 Karl A, Birbaumer N, Lutzenberger W, et al. Reorganization of motor and somatosensory cortex in upper extremity amputees with phantom limb pain. J Neurosci $2001 ; 21: 3609-18$

21 Rijnties $M$, Tegenthoff $M$, Liepert J, et al. Cortical reorganization in patients with facial palsy. Ann Neurol 1997;41:621-30

22 Brasil-Neto JP, Cohen LG, Pascual-Leone A, et al. Rapid reversible modulation of human motor outputs after transient deafferentation of the forearm: a study with transcranial magnetic stimulation. Neurology 1992;42:1302-6.

23 Ziemann U, Wittenberg GF, Cohen LG. Stimulation-induced withinrepresentation and across-representation plasticity in human motor cortex. J Neurosci 2002;22:5563-71.

24 Tsubokawa T, Katayama Y, Yamamoto T, et al. Chronic motor cortex stimulation in patients with thalamic pain. J Neurosurg 1993;78:393-401.

25 Fujii M, Ohmoto Y, Kitahara T, et al. Motor cortex stimulation therapy in patients with thalamic pain. Neurol Surg 1997;25:315-19.

26 Dejerine J, Roussy J. Le syndrome thalamique. Rev Neurol (Paris) 1906;14:521-32.

27 Garcia-Larrea L, Peyron R, Mertens P, et al. Electrical stimulation of motor cortex for pain control: a combined PET-scan and electrophysiological study. Pain 1999;83:259-73.

28 Basbaum Al, Fields HL. Endogenous pain control systems: brainstem spinal pathways and endorphin circuitry. Annu Rev Neurosci 1984;7:309-38.

29 George MS, Wassermann EM, Williams WE, et al. Mood improvements following daily left prefrontal repetitive transcranial magnetic stimulation in patients with depression: a placebo-controlled crossover trial. Am J Psychiatry 1997; 154:1752-6.

30 George MS, Nahas Z, Molloy M, et al. A controlled trial of daily left prefrontal cortex TMS for treating depression. Biol Psychiatry 2000;48:962-70. 